\title{
A New Algorithm of Boolean Function and Its Application
}

\author{
Hong Mei PEI ${ }^{1, a^{*} \text {, Gui Feng HUANG }}{ }^{2, b}$ and Xuan Hai LI ${ }^{3, c}$ \\ ${ }^{1}$ Department of Basis, Dalian Naval Academy, Dalian, China \\ ${ }^{2}$ Department of Basic, Dalian Institute of Science and Technology, Dalian, China \\ ${ }^{3}$ Department of Basis, Dalian Naval Academy, Dalian, China

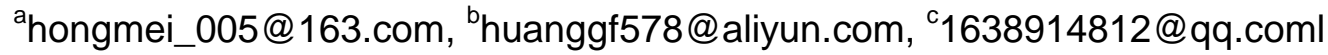

Keywords: Graph Property, Boolean Function, elusive function, Dual Algorithm, Karp conjecture

\begin{abstract}
This article proposes a new algorithm of constructing Boolean function, that is, the dual algorithm, to overcome the drawback of the existing algorithm. Through comparing the two algorithms by calculating a Boolean function of two graph properties, we show the strengths and weakness of the two algorithms.
\end{abstract}

\section{Introduction}

Compared with the normal Boolean functions, the main difference between Boolean functions and graph properties is that graph property doesn't have specific function expression. If we obtain the graph property expression firstly, then we can make a study of the graph property's elusiveness by using the direct method. The traditional algorithm uses all the true assignments to calculate function expression. The traditional algorithms has its weakness, which is that when the numbers of the true assignments are much more greater than the numbers of false assignments, it will have computationally intensive weakness. To overcome this weakness, this article proposes the dual algorithm, which uses all the false assignments to figure out algorithm expression.

\section{Dual Algorithm}

This article proposes a new algorithm named dual algorithm to figure out Boolean function, which is to build Boolean function by using false assignments.

Dual Algorithm: For every false assignment, build boolean sum as below method firstly: if the valuation of variable $x_{i}$ is 0 , then $x_{i}$ will be put in boolean sum. Otherwise, if the valuation of variable $x_{i}$ is 1 , then $\bar{x}_{i}$ will be put in boolean sum. Multiply boolean sum that built by all the false assignments by boolean product, then we will obtain the boolean function's expression that expressed by boolean sum, boolean product and the operation of negation.

Table 1 Functional relationships table

\begin{tabular}{ccc}
\hline$x_{1}$ & $x_{2}$ & $f\left(x_{1}, x_{2}\right)$ \\
\hline 0 & 0 & 0 \\
0 & 1 & 1 \\
1 & 0 & 1 \\
1 & 1 & 0 \\
\hline
\end{tabular}

Based on the assignment of variables and functions values listed in table 1, we will obtain the boolean function by using the traditional algorithm $[1,2]$.

$$
f\left(x_{1}, x_{2}\right)=\bar{x}_{1} x_{2}+x_{1} \bar{x}_{2}
$$


Calculate by using above dual algorithm, we will obtain the below Boolean function:

$$
f\left(x_{1}, x_{2}\right)=\left(x_{1}+x_{2}\right)\left(\bar{x}_{1}+\bar{x}_{2}\right)
$$

However,

$$
f\left(x_{1}, x_{2}\right)=\left(x_{1}+x_{2}\right)\left(\bar{x}_{1}+\bar{x}_{2}\right)=x_{1} \bar{x}_{1}+x_{1} \bar{x}_{2}+x_{2} \bar{x}_{1}+x_{2} \bar{x}_{2}=x_{1} \bar{x}_{2}+x_{2} \bar{x}_{1}
$$

Thus we can find that for the boolean function listed in Table 1, the Eq. 1 calculated by using the traditional algorithm is the same with the equation Eq. 2 that calculated by dual algorithm which we proposed in this article. Then we presume that calculating by dual algorithm will obtain the same result with that calculating by the traditional algorithm under any circumstances. Then we will prove this result we presumed.

Theorem The boolean function obtained by dual algorithm is correct.

Proof To prove the boolean function expression that calculated by dual algorithm is correct, we only need to prove that for any assignment of variables, we will obtain the same Boolean function value with the results listed in the function relationships table.

Let $f\left(x_{1}, x_{2}, \cdots, x_{n}\right)$ be a boolean function with $n$ variables, which is obtained by multiply $k$ boolean sums by boolean product using the dual algorithm. Since each boolean sum is determined by the definition of the dual algorithm, the $k$ boolean sums include all the false assignment terms. Then, for any assignment of variables, we have

(1) If it is a false assignment, then the boolean function has the corresponding boolean sum with it, and the value is 0 , thus the function value is 0 . It is the same with given results.

(2) If it is a true assignment, then the function expression doesn't have the corresponding boolean sum with it, thus all the boolean sums' value in the boolean function are 1. Otherwise, if there exist a boolean sum which value is 0 , according to the definition of the function, this group of assignment is false assignment, which is at odds with our assumption. So the function value is 1 , the same with given results.

Conclusions as a result, for any group of assignment of $n$ variables, the values of boolean function calculated by dual algorithm is always the same with given values, which proves that the dual algorithm is correct.

We have proved the correctness of the dual algorithm. But is it redundant that we propose the dual algorithm while there is already an algorithm exists? Does the dual algorithm itself has practical use? Then we will show how the dual algorithm is indispensable by comparing the dual algorithm with the traditional algorithm.

\section{Comparing Dual Algorithm with Traditional Algorithm}

We will compare dual algorithm with traditional algorithm by using specific example.

Table 2 Coneectedness's functional relationships

\begin{tabular}{ccccccccc}
\hline$x_{12}$ & 0 & 0 & 0 & 0 & 1 & 1 & 1 & 1 \\
\hline$x_{13}$ & 0 & 0 & 1 & 1 & 0 & 0 & 1 & 1 \\
\hline$x_{23}$ & 0 & 1 & 0 & 1 & 0 & 1 & 0 & 1 \\
\hline$f\left(x_{12}, x_{13}, x_{23}\right)$ & 0 & 0 & 0 & 1 & 0 & 1 & 1 & 1 \\
\hline
\end{tabular}


According to the functional relationships of Table 2, by using above two algorithms, we get the boolean function of graph connectedness with 3 vertexes[3].

$$
f\left(x_{12}, x_{13}, x_{23}\right)=x_{12} x_{13}+x_{13} x_{23}+x_{23} x_{12}
$$

And

$$
x_{i j}=\left\{\begin{array}{cc}
1 & \text { graph Ghas edge }(i, j) \\
0 & \text { otherwise }
\end{array}, \quad(i, j=1,2,3)\right.
$$

It is OK to use both the traditional algorithm and dual algorithm to calculate the boolean function of this type of graph property. This is because graphs with 3 vertexes which including isomorphism, have $2^{3}=8$ types in total, that is, have $2^{3}=8$ types of assignments of variables. According to Table 2 , we can figure out that both true assignments and false assignments all have 4 types, thus the amount of calculation is the same while calculating boolean function expression by using any type of algorithms.

Assume a graph of order 4's graph property $P$ as : this graph's edge is no more than 1 . Then the number of true assignments are only 7 , but the number of false assignments are $2^{6}-7=64-7=57$, in this occasion, we can using the traditional algorithm to calculate, that is, calculate the graph property's boolean function by using true assignments, which is easy to implement.

Table 3 Functional relationships

\begin{tabular}{ccccccccc}
\hline$x_{12}$ & 0 & 0 & 0 & 0 & 1 & 1 & 1 & 1 \\
\hline$x_{13}$ & 0 & 0 & 1 & 1 & 0 & 0 & 1 & 1 \\
\hline$x_{23}$ & 0 & 1 & 0 & 1 & 0 & 1 & 0 & 1 \\
\hline$f\left(x_{12}, x_{13}, x_{23}\right)$ & 0 & 1 & 1 & 1 & 1 & 1 & 1 & 1 \\
\hline
\end{tabular}

If a graph of order 3's graph property $P$ is : the graph has at least 1 edge , then this graph property's functional relationships can be expressed as Table 3. It has only one false assignments (when the graph has no edge), but has 7 true assignments. The graph property's boolean function calculated by the dual algorithm as below:

$$
f\left(x_{12}, x_{13}, x_{23}\right)=x_{12}+x_{13}+x_{23}
$$

Because of that in all assignments of the graph property, the number of false assignments is much more smaller than the number of true assignments. If we use the traditional algorithm to calculate this boolean function of the graph property, then not only the calculation amount is a large one, but also the function expression is very complex. That is:

$$
f\left(x_{12}, x_{13}, x_{23}\right)=\bar{x}_{12} \bar{x}_{13} x_{23}+\bar{x}_{12} x_{13} \bar{x}_{23}+\bar{x}_{12} x_{13} x_{23}+x_{12} \bar{x}_{13} \bar{x}_{23}+x_{12} \bar{x}_{13} x_{23}+x_{12} x_{13} \bar{x}_{23}+x_{12} x_{13} x_{23}
$$

Thus the dual algorithm's advantages and practicability can be found.

\section{Conclusion}

According to above boolean functions of graph property's calculating, we can get this conclusion: While calculating a boolean function's expression, choose which algorithm to calculate can have a 
smaller calculation amount is mainly depends on which assignments' number are smaller in all the assignments. That is, while true assignments' number is smaller than that of false assignments', then using traditional algorithm is preferred. While true assignments' number is greater than false assignments' number, then using the dual algorithm that we proposed is preferred, in this occasion, the calculation amount is much more smaller than using the traditional algorithm. This article is just using small order graph's graph properties to prove this conclusion, for higher order graph's graph properties, the two algorithm's characteristic will be shown more obviously. So we can find that this two algorithms oppose each other also complement each other, thus the dual algorithm proposed in this article is indispensable.

\section{Acknowledgements}

This work was supported by the Dalian Naval Academy for Basic Research

\section{References}

[1] Du Ding-zhu, Decision Tree Theory [M]. Hunan Education Press 1998.

[2] Li De-ying, Du Ding-zhu. Graph Properties Based on the Karp Conjecture[J]. Chinese Science Bulletin, 2000: 2129-2134.

[3] Wang Shuhe. Graph Theory[M]. Science Press, 1998.

[4] Best M R, van Emde Boas P, H.W.Lenstra et al. A sharpened version of the Aanderaa-Tosenberg Conjucture, Report ZW 30/74, Mathematisch Centrum Amsterdam 1974.

[5] A. L. Rosenberg, On the time require to recognize peoperties of graphs: A problem, SIGACT News, 5:4(1973) 15-16.

[6] R. L. Rivest, S. Vuillemin, A generalization and proof of the Aanderaa-Rosenberg conjecture, in Proc. SIGACT Conf, Albuquerque, 1975.

[7] R. L. Rivest, S. Vuillemin, On recognizing graph properties from adjacency matrices, Theor. Comp. Sci., 1976, 3:371 384.

[8] S.-X. Gao, X. Hu, W. Wu, Nontrivial monotone weakly symmetric Boolean functions of six variables are elusive, Theoret. Comput. Sci., 1999, 223:193-197.

[9] S.-X. Gao, X. Hu, W. Wu et al, Nontrivial monotone weakly symmetric Boolean functions of ten variables are elusive, J. Comples. 15(1999):526-536.

[10] S.-X. Gao, X. Hu, W. Wu et al, Nontrivial monotone weakly symmetric Boolean functions of twelve variables are elusive, Discrete Math., 253(2002):19-34. 